\title{
ANTROPOMARKETING HOLÍSTICO PARA ORGANIZACIONES INTELIGENTES
}

Carlos Cavani Grau

\section{Resumen}

La Antropología y el Marketing son Ciencias Sociales que poseen un tratado de conocimientos orientados a la objetividad y al descubrimiento de la verdad de la realidad, sin conclusiones axiomáticas, como las Matemáticas. Asimismo constituyen dos Ciencias perfectamente aplicables -de acuerdo a las circunstancias- a la comprensión, análisis, e interpretación de sociedades, mercados e instituciones de la Humanidad.

Antropomarketing para Organizaciones inteligentes afirma que las decisiones de las organizaciones empresariales e institucionales requieren como condición fundamental comprender la naturaleza antropológica de las decisiones de las personas consumidoras, y que sus actos de consumo representan experiencias impregnadas con un sello sociocultural e histórico.

Palabras clave: Sintagma, Neuromarketing, Paradigma, Trademarketing, Sinergia, Cognición, Holismo, Industria Osteoodonqueratica, Antropología, Economia, Psicoanálisis, Psicología Social.

\footnotetext{
Abstract

Anthropology and Marketing are Social Sciences that have a treaty of knowledge oriented to the objectivity and the discovery of the truth of reality, without axiomatic conclusions, like Mathematics. Also they constitute two Sciences perfectly applicable -in agreement to the circumstances-, the understanding, analysis, and interpretation of societies, markets and institutions of the Humanity.
} 
Antropomarketing for Intelligent Organizations affirms that the decisions of the enterprise and institutional organizations require as a fundamental condition fundamental to include/understand the anthropological nature of the decisions of the consuming people, and who their acts of consumption represent experiences impregnated with a sociocultural and historical seal.

Key words: Sintagma, Neuromarketing, Paradigm, Trademarketing, Sinergia, Cognition, Holismo, Osteoodonqueratica Industry, Anthropology, Economy, Psychoanalysis, Social Psychology.

\section{INTRODUCCIÓN}

La primera impresión que podría provocar la noción Antropomarketing holístico sería de curiosidad, o de perplejidad, o solo de sorpresa por el sentido de la palabra: no solo es Antropomarketing sino, además, holístico. La intención de este ensayo es intentar explicar cuál es su verdadera naturaleza y los fundamentos que le dan contenido a una nueva disciplina del marketing en las actuales circunstancias, ciencia e instrumento clave para la gestión de las Organizaciones inteligentes.

Estas organizaciones a las que aspiramos, deben ser proactivas, es decir, no deben dejarse vencer por las circunstancias y tienen la capacidad de adelantarse al impacto -a veces aniquilador- de los imprevistos. Asimismo, estas organizaciones poseen un complejo poder de resiliencia para recuperarse de los fracaso, que son inminentes, y ser admirados por sus clientes y la sociedad.

\section{ANTROPOLOGÍA E HISTORIA PARA COMPRENDER NUESTRAS DECI- SIONES DE CONSUMO}

Los primeros hominidos registrados por importantes estudios arqueo- lógicos y antropológicos, como los Australopithecus en todas sus variantes, los Homo Ergáster, los pitecántropus erectus, los hombres de Neanderthal, los homínidos de Java o los primeros homohabilis, fueron eminentemente cazadores, con una clara división del trabajo y responsabilidad en la constitución y supervivencia de los primeros clanes u organizaciones nómades. En el caso de las mujeres, estas fueron paulatinamente especializándose en las tareas de recolección, selección y preservación de los productos de supervivencia, los alimentos, así como también empezaron con el cuidado de los hijos del clan y su supervivencia como especie. La permanencia consuetudinaria de la mujer en sus refugios precarios, fue creando en ellas un conjunto de patrones antropográficos, como la comunicación, la observación de los productos de supervivencia, y otras especialidades paulatinamente más complejas.

Camilo Herrera (s/f), sostiene que el hombre ha sido cazador y la mujer recolectora por más de 5.000 años, y que los últimos 100 años de transformaciones no han podido cambiar ese proceso, solo lo han modificado. El hombre puede estar en casa siendo clásico en 
su forma de vestir, manteniendo otros patrones comportamentales, porque como cazador no quiere ser llamativo; mientras que la mujer, al salir al mundo laboral, posee como principal arma de caza ser atractiva, como lo hacía anteriormente para llamar la atención del cazador, en la búsqueda paciente pero perseverante de seguridad.

Pero aún son muchas más las mujeres trabajadoras que actualmente miran la fecha de vencimiento de los productos, que es una inclinación antropológica, lo cual pasan por alto los hombres que comienzan a hacer el mercado para la casa. Es muy probable que a los jóvenes adultos aún no les guste comprar productos de primera necesidad o abarrotes en los supermercados o mercadillos -esa función se la dejan a los padres-, pero el modelo paterno, tarde o temprano, los influenciará.

Así, el mercadeo y el trademarketing tienen que entrar en los terrenos pedregosos de aceptar que los seres humanos tenemos una historia que guía muchos de nuestros comportamientos. A esto, algunos estudiosos lo han querido llamar instintos o, incluso, neuromarketing, que no es otra cosa que el sentido común de reconocer que somos parte de un proceso antropológico e histórico, como sostiene con coherencia Camilo Herrera.

Es casi imposible encontrar un comportamiento del consumidor que no tenga vínculos antropológicos e históricos. Lo que ocurre es que nuestra capacidad de adaptación es tan veloz que dejamos atrás inventos fun- damentales simplemente porque hay algo mejor o más moderno; pero en el momento más inesperado usamos el celular de última generación para escribir notas como hacemos en un cuaderno, y chateamos como si pusiéramos telegramas.

Nos es imposible desvincularnos de los procesos anteriores, porque toda solución parte de una necesidad insatisfecha, o un deseo insatisfecho parcial o completamente. Claro que esto sucede con los consumidores de vanguardia en mayor medida que con los consumidores intermedios, o mucho más que los rezagados.

Por ejemplo, la empresa Apple innova permanentemente y lanza sus nuevos productos con shows mundiales, como el reciente Ipad, y congrega a nivel global a sus fans tecnológicos, que son consumidores de vanguardia ávidos de tener en sus manos el nuevo "esperpento tecnológico". Estos se amanecen en la acera esperando que las tiendas de productos electrónicos se abran para abalanzarse y adquirir uno, como el Ipad que solo fue lanzado el año pasado pero que ha sumado en esta nueva versión otras innovaciones respecto al anterior.

\section{LA NOCIÓN HOLÍsTICA}

La noción Holística proviene de holismo (Wikipedia, 2013) (del griego tos [holos]; todo, entero, total), idea de que todas las propiedades de un sistema dado (por ejemplo, biológico, químico, social, económico, mental o lingüístico), no pueden ser determinados o 
explicados por las partes que los componen. El sistema como un todo determina cómo se comportan las partes. Como adjetivo, holístico significa una integración total frente a un concepto o situación.

El principio general del holismo fue resumido por Aristóteles en la Metafísica (libro que escribió después de la Física): "El todo es mayor que la suma de sus partes".

Se puede definir como el tratamiento de un tema que implica relaciones de todos sus componentes, aun cuando muchas veces son invisibles. Se usa como una tercera vía o un nuevo enfoque a un problema. El holismo enfatiza la importancia del todo, que es más grande que la suma de las partes (propiedad de sinergia), y da importancia a la interdependencia de estas.

Trata de presentarse directamente como un axioma y no como una hipótesis. La validación es su principal problema, en cuanto asume las propiedades del método científico.

La holística es, por tanto, aquello perteneciente al holismo, una tendencia o corriente que analiza los eventos desde el punto de vista de las múltiples interacciones que los caracterizan. El holismo supone que todas las propiedades de un sistema no pueden ser determinadas o explicadas como la suma de sus componentes.

\section{UNA REPRESENTACIÓN DEL HOLISMO}

El holismo alude a contextos y complejidades que entran en relación, ya que es dinámico. Para la comprensión holística, el todo y cada una de las partes se encuentran ligadas con interacciones constantes. Por eso, cada acontecer está relacionado con otros acontecimientos, que producen entre sí nuevas relaciones y eventos en un proceso que compromete el todo.

La comprensión de los procesos y las situaciones debe tener lugar desde el propio holos, ya que en su dinamismo, surge una nueva sinergia, ocurren nuevas relaciones y se generan nuevos acontecimientos. Por lo tanto, el todo es lo determinante, aun cuando este reconocimiento no impide que se analice cada caso en particular.

La perspectiva holística implica una superación de los paradigmas para propiciar la figura del sintagma, entendida como una integración de paradigmas. Una actitud sintagmática supone la convergencia de diversas perspectivas, lo que solo puede lograrse con criterios holísticos.

\section{FUNDAMENTOS}

En consecuencia, el Antropomarketing Holístico constituye un todo, un sistema compuesto por un conjunto de elementos que son a su vez sus propios fundamentos endógenos, como las necesidades y los deseos del ser humano; los patrones comportamentales, como la personalidad, las motivaciones y las actitudes; los patrones cognitivos, como el aprendizaje. También, otros patrones antropográficos con influencia de Marketing, como el consumo y la comunicación, y otros 
influenciados por la posmodernidad, como los estilos de vida y patrones neurales, como las sensaciones y percepciones. De igual manera, como los patrones exógenos representados por la cultura, la familia, los grupos de referencia y las clases sociales. Estas nociones las trataremos más adelante.

\section{ECONOMÍA, SOCIOLOGÍA Y PSICO- LOGÍA INDIVIDUAL Y SOCIAL, LAS OTRAS FUENTES DEL MARKETING}

Además de la antropología, las otras fuentes teóricas del marketing contemporáneo son la economía, la sociología y la psicología individual y social. La Economía, como ciencia social, no solo es la que explica la utilización de los recursos escasos para el desarrollo sustentable del proceso económico de los pueblos, sino el fundamento poderoso del intercambio basado en la ley de la oferta y la demanda.

Adam Smith, precursor de la economía, sostuvo en su texto La Rique$z a$ de las naciones (The wealth of the Nations, 1776) que las sociedades no son solidarias y altruista per se, sino que la oferta y la demanda generan las condiciones para el funcionamiento del proceso económico.

Pero la correspondencia entre el marketing y la economía reside en que el primero es capaz -orientado correctamente por sus operadores- de estimular los términos de intercambio a través de un producto adecuado, un precio razonable, una plaza o canal de distribución conveniente y una promoción oportuna.
Otros aportes de la economía al marketing son el concepto de homo economicus, aquel que siempre consumirá el producto que le dé más utilidad en función del precio, pero que luego de sufragar sus gastos elementales es capaz de exigirse al máximo para construir un ahorro que le permita cierta gratificación por el sacrificio realizado para sobrevivir.

Los economistas lo llaman los costos marginales. La ley de Lancaster, que sostiene que no es el producto como tal el que genera la satisfacción, sino los atributos que lo caracterizan. Dubois y Rovira (1998) dicen que no es en la pasta de dientes en sí misma donde está el origen de la satisfacción (o de la insatisfacción) del consumidor, sino en el hecho que este producto procure un mejor aliento, protección contra la caries, los dientes más blancos, etc.

Las elecciones de los individuos se basan en las características y subcaracteristicas de los productos, antes que en las entidades-productos.

Por ello, reiteramos que existe una concepción económica del marketing que termina por reconocer que contemporáneamente hablando, la disciplina es probable y posible en una economía de mercado, y cuando la oferta supera a la demanda. Es que el marketing es capaz de estimular los términos microeconómicos del intercambio, la oferta y la demanda. Algún autor reconoce al marketing como la ciencia del intercambio.

En términos del proceso económico, las fases sucesivas de Extracción, 
Transformación, Distribución y Consumo $^{6}$, son claves para comprender que el objetivo fundamental del proceso es la creación del valor agregado, que el papel del marketing es condicionante en las primeras etapas y determinante en la etapa de la distribución, en la que es capaz de construir su propia cadena de valor basado en la satisfacción irrestricta de las necesidades y deseos del consumidor. Nos referimos a las utilidades de forma, de lugar, de tiempo, y de posesión.

\section{CONSUMO}

El consumo es una actividad humana omnipresente, porque se representa en una constelación de actividades humanas.

Es la fase en la que el cliente consumidor ha obtenido el producto y lo ha usado o consumido, o lo ha desechado.

¿Por qué compra la gente? Esta pregunta sigue devanando los sesos de los especialistas, quienes continúan respondiéndola para nuestra felicidad o mayor confusión desde diferentes ópticas: la económica, la sociológica, la antropológica o la conductual. Los mercadólogos, cual pragmáticos observadores y decisores de las campañas comerciales, las adaptan dramáticamente a los designios de los consumidores.

Pero, ¿cómo han cambiado los consumidores peruanos y latinoamericanos en los últimos años? Cualquier respuesta inmediatista se estrellaría con la realidad. Hasta hace algunos años -utilizando un argot marketero- eran "lornas", pero ahora se han convertido en verdaderos "tiburones", por ansiosos, recelosos, desconfiados, desafiantes y compulsivos.

Intentando ofrecer algunas explicaciones a la gran pregunta: ¿por qué compra la gente?, encontramos que las necesidades y los deseos humanos en pos de satisfactores múltiples e indistintos están en el centro de la Motivación humana. Por qué compra la gente-curiosamente el título de uno de los videos precursores del Marketing más populares de la década del setenta-, es una pregunta clave para entender las razones fundamentales de las decisiones de compra de las personas, las mismas que por lo general están orientadas a un consumo que Henry Assael (2005) define como un acto omnipresente tan vital que en el acto de dormir que representa consumir oxígeno y tan superfluo o banal como comprar un Ferrari de 250,000 dólares. Y si científicamente respirar es un acto de consumo para vivir; comprar el Ferrari solamente constituiria un hito extraordinario... en el ego de su cliente propietario.

Sea por cuesiones vitales o banales, las personas-consumidoras tienen millones de razones para comprar, como millones de razones para no hacerlo; y cuanto más estudiamos tales razones, más ignoramos las verdaderas causas que los llevaron a esas decisiones de compra.

Del conjunto de teorias explicativas de las razones de compra del consumidor, sean económicas, sociológicas, 
del aprendizaje, conductuales o psicológicas; esta última es particularmente importante -sin menoscabo de las anteriores- porque se funda en la existencia de actitudes cognitivas o racionales, emotivas, afectivas e inconscientes, todas ellas razones relativas, porque las actitudes no son patrones comportamentales fácilmente observables (Chaparro, 2008).

Las necesidades fisiológicas y de seguridad representan poderosas necesidades innatas, como el hambre o el sueño o la comunicación. Las razones emotivas son de naturaleza psicógena y reactivas a eventos emocionales de impacto, como el nacimiento de un bebe; en tanto que las motivaciones afectivas, también de naturaleza psicógena, advierten relaciones de pertenencia de profunda naturaleza de afiliación, como la inclinación gregaria. Razones inconscientes conducen a adquirir satisfactores inéditos, que alteran la conducta normal de compra, debido a la presión de las formas de ventas por impulso (degustaciones, óbolos inopinados, colectas públicas, etc.).

Repasando las interpretaciones de las teorías de consumo, se concluye que la teoría económica del consumo está representada por el concepto de "homo economicus", aquel que siempre consumirá el producto que le dé más utilidad en función del precio, el hombre económico que luego de sufragar sus gastos fundamentales de sobrevivencia orienta el saldo económico que le sobra a decisiones de consumo más bien placenteras, como premiándose por los estados de privación ex- perimentadas en el contexto de una sociedad de consumo.

Los economistas llaman a estas decisiones "costos marginales", los cuales grafican a la comunidad de consumidores tomando decisiones de consumo austeras, sin brillo, en un escenario económico en el que la demanda supera a la oferta.

La Sociología constituye, como ciencia social, el estudio de las relaciones interpersonales y su vinculación con el entorno.

La teoría sociológica se funda en la preminencia de los grupos sociales sobre el individuo, estos constituyen una suerte de hermano mayor que circunda, influencia y lo presiona a la búsqueda, indagación y compra de satisfactores, que a la postre solo fortalecerán el espíritu gregario sobre el espíritu individual. El individuo solo desea pertenecer al grupo de referencia y satisfacer una profunda necesidad de refugio social: es el individualismocolectivismo, en el paralelo histórico y genial de Geert Hofstede, que propone la paulatina y accidentada comprensión de que nuestros escasos espacios personales van reduciéndose por la presión de las sociedades posmodernas y de la globalización.

La teoría del aprendizaje se basa en la certeza del positivismo de las experiencias acumuladas a través del tiempo por sobre la obtención de nuevos conocimientos y experiencias de vida, de consumo.

Y en atención a las necesidades, Abraham Maslow, a través de la Teoría de las Necesidades o Pirámide de las 
Necesidades, identifica dos grandes grupos de necesidades:

1. Necesidades Primarias o Innatas (llamadas también de mantenimiento o deficitarias).

2. Necesidades Secundarias o adquiridas (llamadas también de crecimiento, o meta desarrollo, o psicógenas)

Las necesidades primarias o innatas, son:

a) Fisiológicas

Están relacionadas a las necesidades de supervivencia, como alimentación, vestido, vivienda, maternidad, sexo.

\section{b) Seguridad}

Están relacionadas a la necesidad de seguridad y orden, como tener orden en la vida, vivir libre de riesgos, tener seguridad tanto a nivel físico como emocional. En la acepción de Hofstede (Stephen Robbins, 1998), una forma de vida alejada de la inseguridad con un refugio asegurado. Se manifiesta asimismo como un fuerte instinto de supervivencia.

Las necesidades secundarias o adquiridas (crecimiento o meta desarro1lo), son:

\section{a) De afiliación o sociales}

Amor, aprecio, comprensión, cariño y pertenencia a grupos (proporcionan también sentido de seguridad al individuo). Asociación a clubes, agrupaciones civiles, grupos de estudio.

b) Estima o Reconocimiento

Un tributo de Maslow al extraordinario aporte de Sigmund Freud (1932) [3] respecto al desarrollo de la personalidad humana expuesta a través del Ibíd. o Id, ego, y superego, nociones señeras y complejas planteadas por el creador del Psicoanálisis. El ego cifrado en el orgullo propio y expresado en múltiples formas por las personas como la consecución de un status, o un rol societario, niveles de prestigio e imagen ante otros grupos de referencia.

c) Autorealización o autosuperación

Es el grado más alto de la Jerarquía de las necesidades humanas, donde en teoria, la persona ha satisfecho todas sus necesidades. En teoria, parafraseando a David MacLelland (1970), porque a través de las necesidades de autorrealización aun busca mayores logros, mayores reconocimientos, o un mayor poder. Otra lectura de estas necesidades representa al individuo orientado a trascender, dejar huella, dejar un legado, expresar sus ideas y convicciones. La filantropía y la continua búsqueda de aporte a la sociedad podrian ser otra representación de la autorrealización.

De acuerdo a Abraham Maslow, cuando las necesidades de orden inferior son satisfechas, se va activando o generando en el individuo otro tipo de necesidades. Ejemplo: Si la persona cree que morirá de hambre, buscará incansablemente la forma de conseguir alimento (necesidades fisiológicas). Cuando ya logró conseguir alimento, buscará la forma de asegurar ese alimento a través de la búsqueda de un trabajo seguro que le permitirá asegurar dicho alimento, etc.

Sin embargo, existen algunos detractores a esta teoría, que postulan que no todos los individuos satisfacen sus necesidades en este orden, o que 
inclusive, tienen las mismas necesidades. Ejemplo: Una persona de escasos de recursos económicos buscará la forma de poder conseguir alimento para buscar satisfacer sus necesidades de orden inferior. En contraposición, una persona solvente económicamente posiblemente ya se encuentre en el más alto escalafón de esta Pirámide, porque el dinero ya le ha permitido satisfacer todas sus necesidades anteriores.

La Psicología trata de la conducta del hombre, de sus experiencias intimas, y de las relaciones entre ambas. También se ocupa de los órganos que ejercen influencia sobre la experiencia y el comportamiento y de las conexiones de estos con el ambiente (Werner Wolf, 1953).

La relación de la Psicología con el marketing está vinculada a los temas de la conducta del consumidor y al posicionamiento, entendido como la conquista de la mente del mismo consumidor.

Trout y Ries, legendarios maestros del marketing, afirmaron en la década del setenta que el marketing no es una batalla de productos sino una batalla de percepciones. Con este argumento dieron un giro drástico en la evolución del marketing contemporáneo, obligando a los especialistas a volver sus ojos sobre las fuentes del marketing: los consumidores.

Es que la personalidad, los estilos de vida, las motivaciones, las actitu- des, las sensaciones, las percepciones y los patrones cognitivos (aprendizaje) constituyen los patrones comportamentales relevantes -aunque no son los únicos- que delinean endógenamente el comportamiento del consumidor. Los modelos contemporáneos de la toma de decisiones de consumo, como Nicosia, Shiffman, Engel-Blackwell-Kollat, rescatan el conjunto de patrones endógenos comportamentales así como los patrones exógenos de naturaleza social y antropográfica, como la cultura, las clases sociales, los grupos de referencia y la familia.

\section{CONCLUSIONES}

Un Antropólogo hace Marketing en las Organizaciones Inteligentes, investigando al consumidor y a las personas, sus estilos de vida (actividades, expectativas, intereses, opiniones), su cosmovisión (ciudadanía, interrelaciones, felicidad), su genealogia (historia, procedencia, raíces, arraigo-desarraigo), y sus aspiraciones (logros, poder, filantropía, hedonismo, altruismo, autorrealización). Por su parte, un Mercadólogo hace Antropología investigando, descifrando, e interpretando las necesidades articuladas y no articuladas de la persona consumidora, que es una tarea enorme y compleja con el propósito de ofrecerles satisfactores de valor para volverlas felices. 


\section{REFERENCIAS BIBLIOGRÁFICAS}

Arellano Cueva, Rolando. (2010). Marketing: Enfoque América Latina. Ed. Pearson.

Assael, Henry. (1998). Conducta del Consumidor. Thomson Editores.

Barrón, Josefina. (2012). Yo no soy tu. Ed. Planeta Perú S.A.

Bell, Genevieve. (2011). Intel's cultural anthropologist. CNN Money- Fortune.

Cavani Grau, Carlos. (2012). Antropomarketing Holístico: Naturaleza y Fundamentos. Revista Horizonte Empresarial. FACEE URP.

Dubois, Bernard \& Rovira, Alex. (1998). Comportamiento del Consumidor. Ed. Prentice Hall.

Freud, Sigmund. (1981). Obras Completas. Ed. Biblioteca Nueva.

Grande, Ildefonso. (2003). Marketing Croscultural, ESIC Editorial.

Iriarte Brenner, Francisco. (2000). Antropología. Fondo Editorial - Textos Universitarios, Rectorado UPIGV.

Rodríguez, Gustavo \& Venturo, Sandro. (2009). Ampay Mujer! Editorial Santillana.

Schiffman, León \& Kanuk, Lazar. (2001).Conducta del Consumidor. Ed. Pearson.

Silva Santisteban, Luis. (2000). Antropología. Fondo Editorial de la Universidad de Lima.

Wolf, Werner. (1953). Psicología. Introducción a la Psicología. Breviarios del Fondo de Cultura Económica, Reed. 1970.

\section{WEBGRAFIA DE LA LECTURA SELECCIONADA}

Agrivalca R.; Canelón S. acanelon@gmail.com / acanelon@ucab.edu.ve

Alcaide, Carlos. www.jcalcaide.com/general/antropologia-y -marketing/

Anthropology Inc. The Atlantic, March 2013.

Antrial.wordpres.com/.../

Herrera, Camilo. www.google.com.

Loudon, Michael; Della Bitta, Albert. www.google.com.

Roca, Jordi y Girona. ¿'Antropólogos en la empresa?: a propósito de la (mal) llamada cultura de empresa". Antrial.wordpres.com/.../ www.wikipedia.com. 